\title{
Pediatric Supracondylar Humerus Fractures: Are Medial Pins Indicated?
}

\author{
Bobby Dezfuli1 ${ }^{*}$, Christopher Larkins' ${ }^{2}$, John T. Ruth', Lisa M. Truchan1 \\ ${ }^{1}$ Department of Orthopaedic Surgery, University of Arizona Medical Center, Tucson, AZ, USA \\ ${ }^{2}$ Department of Orthopaedic Surgery, University of Texas Health Science Center, San Antonio, TX, USA \\ Email: [bdezfuli@ortho.arizona.edu
}

Received 9 April 2014; revised 13 May 2014; accepted 20 May 2014

Copyright (C 2014 by authors and Scientific Research Publishing Inc.

This work is licensed under the Creative Commons Attribution International License (CC BY). http://creativecommons.org/licenses/by/4.0/

(c) (7) Open Access

\section{Abstract}

Background: Pediatric supracondylar humerus fractures are the most common elbow fractures in children. Operative management includes closed reduction and placement of 2 to 3 laterally based pins. Occasionally, a medial pin is used to create a crossed fixation pattern, despite risk of nearly $10 \%$ iatrogenic ulnar nerve injury. The objective of this study was to assess the trends and outcomes in the operative management of pediatric supracondylar humerus fractures at a level one academic trauma center. Materials \& Methods: A retrospective review was performed on all children sustaining a Gartland type II or III supracondylar humerus fractures treated by closed or open reduction and percutaneous pinning in 2006-2008 and 2009-2011 at a level one academic trauma center by two of the authors (JTR, LMT). Pin placement patterns were evaluated and compared based on year performed. Outcomes measured were rates of ulnar nerve symptoms, non-union, re-operation, and varus malalignment. Data analysis was performed using a Fisher exact test on STATA software. Results: A total of 49 patients met inclusion criteria. of 22 patients treated in 2006-2008, 5 (23\%) were type II and 17 (77\%) were type III. From 2009-2011, 16 (59\%) were type II and $11(41 \%)$ were type III. Comparison of pinning pattern in type II fractures between 2006-2008 and 2009-2011 did not indicate statistical significance $(p=0.429)$. Comparison of pinning pattern in type III fractures during the same time period did show that there was a statistically significant decrease $(p=0.010)$ in the number of cross pin fixations. There were no ulnar nerve injuries, non-unions, re-operations, or varus malalignment in any patient on final follow-up. Conclusion: This study shows that there has been a significant decrease in cross pin fixation for pediatric type III supracondylar humerus fractures with equivalent clinical outcomes at a Level I trauma center. Furthermore, performing lateral pinning for type III fractures has eliminated the risk of iatrogenic ulnar nerve injury. Level of Evidence: Level III-Retrospective cohort study.

\footnotetext{
${ }^{*}$ Corresponding author.
} 


\section{Keywords}

\section{Supracondylar Humerus Fracture, Pin Fixation, Ulnar Nerve Injury, Biomechanical Stability}

\section{Introduction}

The most common elbow fractures that are seen in children are the supracondylar fractures of the humerus that can be managed by both operative and non-operative modalities [1]. Whether a supracondylar fracture can be managed non-operatively depends on fracture stability, the degree of displacement and the ability to control displacement through traction [2] [3]. Operative management is managed initially with lateral pinning with further assessment of stabilization in order to determine if a medial pin is needed. Several studies have noted that cross pinning is biomechanically superior to lateral pinning [4] [5]. The drawback with cross pinning is potential iatrogenic ulnar nerve injury [6] [7].

A systematic review in 2007 was conducted due to debates on whether or not there was an increased risk for iatrogenic nerve injury due to medial pinning [7]. The systematic review revealed that although medial and lateral pinning reduced the probability of deformity or loss of reduction by 0.58 times as compared to only lateral pinning, the trade-off was an increased probability of ulnar nerve injury that was 5.04 times higher in cross pinning as compared with only lateral pinning of supracondylar humeral fractures [7]. Due to the findings of this systematic review, we analyzed two board certified fellowship trained orthopedic trauma surgeons (J.T.R. and L.M.T.) at a Level One Trauma Center in order to evaluate trends in pin placement for all children that were treated operatively for a supracondylar humerus fracture.

\section{Materials and Methods}

A retrospective review was performed on all children sustaining a Gartland type II and III supracondylar distal humerus fractures treated by closed reduction and percutaneous pinning between January 2006 to December 2008 and January 2009 to December 2011. Prior to 2009, medial pins were routinely used for type III fractures. At the end of 2008 and beginning of 2009, there was a systematic effort to minimize the use of medial pins. While outcomes until then were good, the concern of iatrogenic ulnar nerve injury persisted. The protocol initiated for surgical technique included the placement of two lateral pins after reduction was obtained. Then, the fracture was stressed and analyzed radiographically. If the fracture was stable, a third lateral pin was placed. If there was motion at the fracture site, then a medial pin was placed. The attending surgeon made this determination.

Patients were excluded if radiographs or post-operative reports could not be obtained to verify the number and placement of Kirschner Wires. Minimum follow-up of 3 months was required. Each chart and postoperative report was carefully reviewed to evaluate patient demographics, intra-operative technique, and pin placement that was verified by radiographs. Charts reviewed from 2006 through 2008 were then compiled and compared with patient charts reviewed from 2009 through 2011.

Two trauma surgeons at a Level One University Trauma Center operatively treated a total of 49 patients from the years of 2006 through 2011. Of these patients, 22 were treated in 2006-2008 and 27 were treated in 20092011. Of the 22 patients treated between 2006 and 2007, 5 (23\%) supracondylar fractures were classified as type II and 17 (77\%) were classified as type III fractures. In 2009-2011 16 (59\%) fractures were classified as type II and $11(41 \%)$ fractures were classified as type III. The number and configuration of pins that were placed per patient in 2006-2008 was compared to the number and configuration of pins that were placed per patient in 2009-2011. In addition to the number of pins placed, the location (medial vs. lateral) of pin placement was compared between the same set of years. Post-operative complications of non-union, varus misalignment deformity assessed by Bauman's angle $>80^{\circ}$, any re-operation, and any ulnar nerve deficits on follow-up were evaluated. IRB approval was obtained prior to start of this study. Data analysis was performed using a Fischer exact test using STATA software.

\section{Results}

A total of 49 patients met inclusion criteria. Of 22 patients treated in 2006-2008, 5 (23\%) were type II and 17 
(77\%) were type III (Table 1). In 2009-2011, 16 (59\%) were type II and 11 (41\%) were type III (Table 1). Figure 1 and Figure 2 show pin placement patterns for 2006-2008 and 2009-2011. Of type II fractures treated in 2006-2008, 4 (80\%) were repaired with lateral only pinning and 1 (20\%) was repaired with both medial and lateral pinning (Table 2, Figure 3). Of the type II fractures repaired in 2009-2011, 15 (94\%) were repaired with lateral only pinning and 1 (6\%) repaired with both medial and lateral pinning (Table 2, Figure 4). Of the type III fractures repaired in 2006-2008, 2 (12\%) were repaired with lateral only pinning and 15 (88\%) repaired with both medial and lateral pinning (Table 3, Figure 5). Of the type III fractures repaired in 2009-2011, 7 (64\%) were repaired with lateral only pinning and 4 (26\%) repaired with both medial and lateral pinning (Table 3, Figure 6). Comparison of pinning pattern in type II fractures between 2006-2008 and 2009-2011 did not indicate statistical significance $(\mathrm{p}=0.429)$. Comparison of pinning pattern in type III fractures during the same time period did show that there was a statistically significant decrease $(p=0.010)$ in the number of cross pin fixation. There were no non-unions, re-operations, ulnar nerve injury, or varus malalignment in any patient on final follow-up.

Table 1. Summary of fracture pattern, pin placement, and outcomes.

\begin{tabular}{ccc}
\hline & $2006-2008$ & $2009-2011$ \\
\hline Total Number of Fractures & 22 & 27 \\
Type II & 6 & 16 \\
Type III & 17 & 11 \\
2 Total Pins & 9 & 14 \\
3 Total Pins & 12 & 13 \\
4 Total Pins & 1 & 0 \\
Ulnar Nerve Symptoms & 0 & 0 \\
Baumann's Angle $>$ 80 & 0 & 0 \\
Re-Operation & 0 & 0 \\
Non-Union & 0 & 0 \\
\hline
\end{tabular}

Table 2. Type II fracture pin pattern.

\begin{tabular}{ccccc}
\hline & \multicolumn{2}{c}{$2006-2008$} & \multicolumn{2}{c}{$2009-2011$} \\
\cline { 2 - 4 } Type II Fractures & $\#$ & $\%$ & $\#$ & $\%$ \\
Number of Fractures & 5 & & 16 & 0 \\
1 Medial Pin and 1 Lateral Pin & 1 & 20 & 1 & 75 \\
1 Medial Pin and 2 Lateral Pins & 0 & 0 & 12 & 19 \\
2 Lateral Pins & 4 & 80 & 3 & 6 \\
\hline
\end{tabular}

Table 3. Type III fracture pin pattern.

\begin{tabular}{ccccc}
\hline Type III Fractures & $2006-2008$ & $2009-2011$ & $2006-2008$ & $2009-2011$ \\
\cline { 2 - 5 } Number of Fractures & $\#$ & $\%$ & $\#$ & $\%$ \\
\hline 1 Medial Pin and 1 Lateral Pin & 17 & 12 & 1 & 9 \\
1 Medial Pin and 2 Lateral Pins & 12 & 70 & 3 & 0 \\
1 Medial Pin and 3 Lateral Pins & 1 & 6 & 0 & 9 \\
2 Lateral Pins & 2 & 12 & 1 & 55 \\
3 Lateral Pins & 0 & 0 & 6 &
\end{tabular}




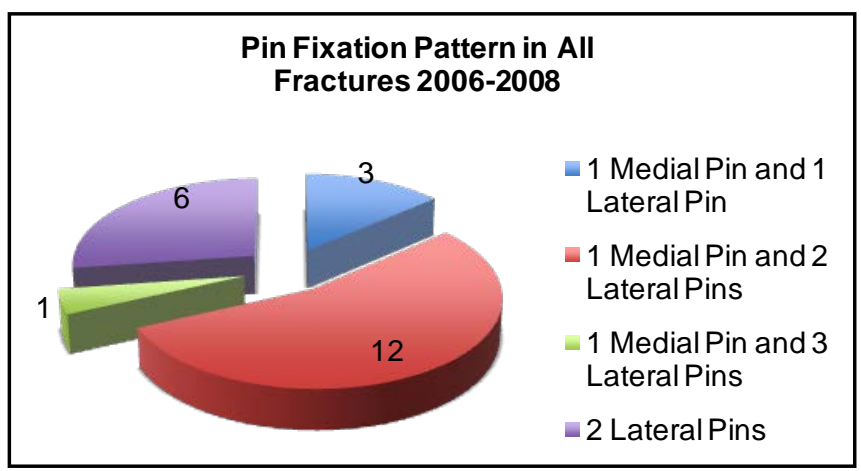

Figure 1. Pin fixation pattern in all fractures 2006-2008.

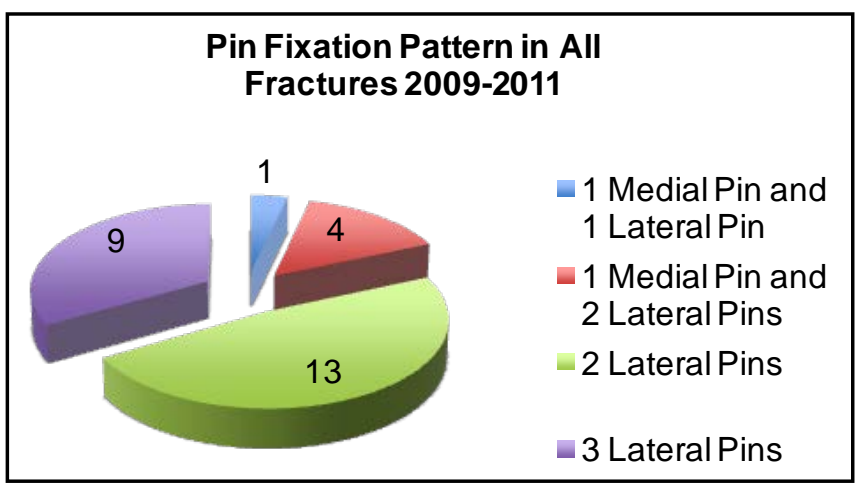

Figure 2. Pin fixation pattern in all fractures.

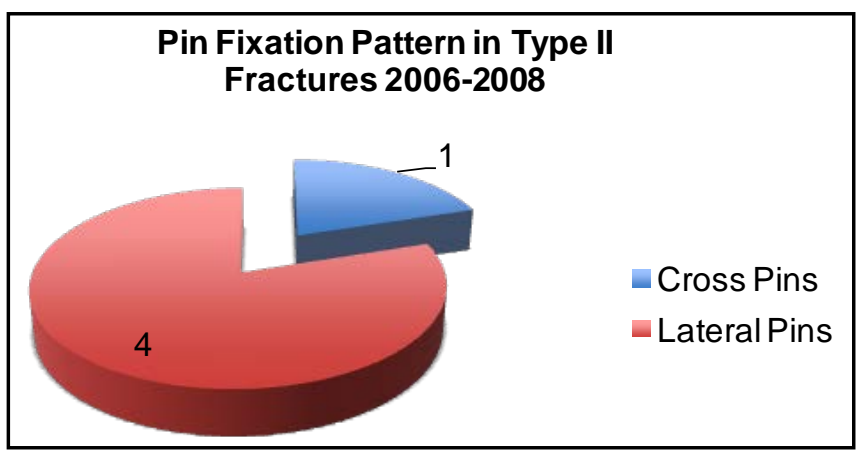

Figure 3. Type II Pin Fixation Pattern 2006-2008.

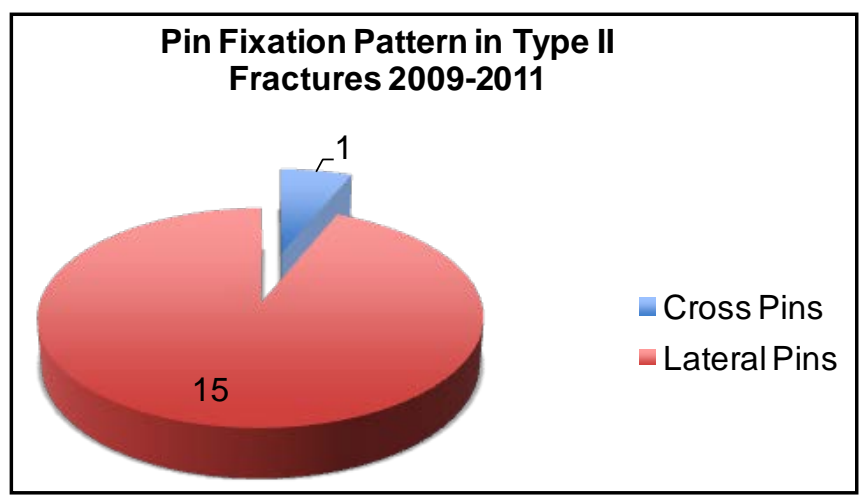

Figure 4. Type II Pin Fixation Pattern 2009-2011. 


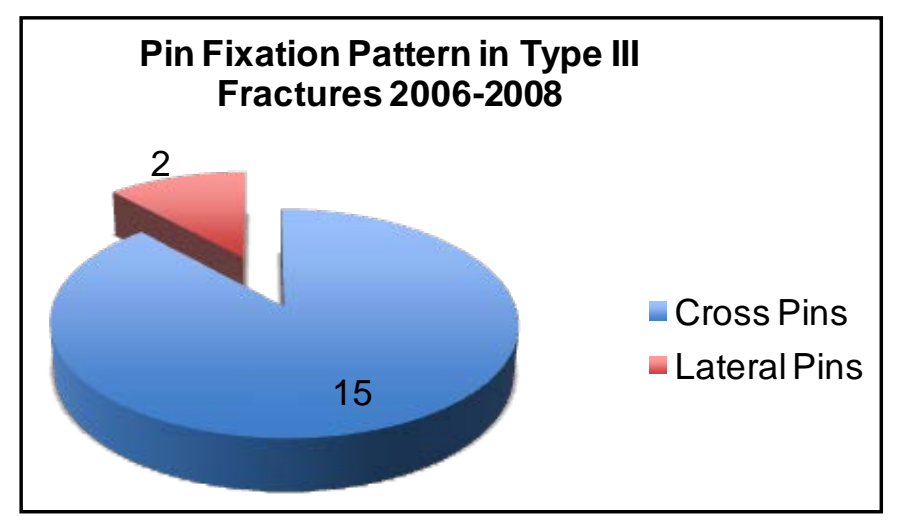

Figure 5. Type III Pin Fixation Pattern 2006-2008.

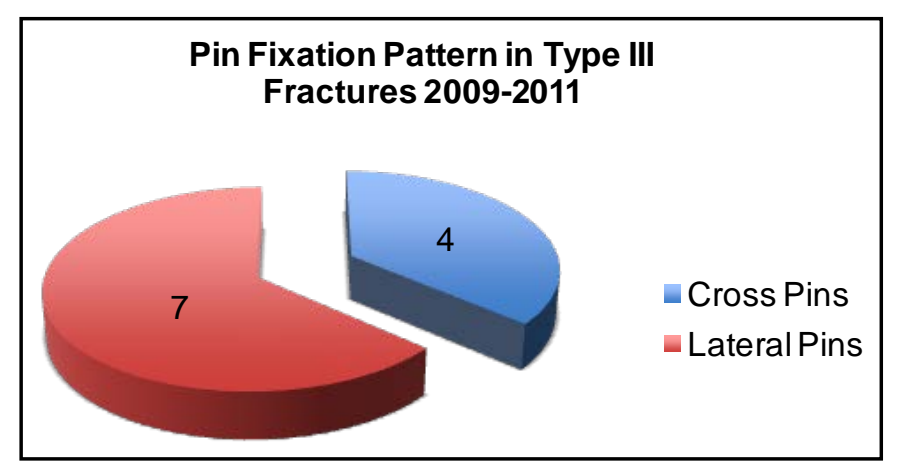

Figure 6. Type III Pin Fixation Pattern 2009-2011.

\section{Discussion}

This study presents a shift in operative management with a significant decrease in cross pin fixation for type III fractures with clinical outcomes, such as ulnar nerve injury, non-unions, re-operation rate, and varus malalignment, comparable to lateral only pinning. The primary advantage of utilizing only lateral pinning to correct supracondylar humeral fractures is to decrease the risk of iatrogenic nerve injury.

Iatrogenic nerve injury often occurs with the placement of a medial pin and can occur after a correctly placed medial pin. Brown and Zinar reported that even with a medial pin that is placed correctly, and there is a risk of damaging the ulnar nerve [8]. By only using lateral pins to fixate supracondylar humeral fractures, there is little risk for iatrogenic injury to the ulnar nerve. Can the authors quantify this risk? The risk was noted in lines 144 and 81. Bronwyn et al. found that there is an iatrogenic ulnar nerve injury for every 28 patients treated with cross pinning as opposed to lateral pinning [9]. Even with the decreased risk for iatrogenic nerve injury, there are disadvantages to using only lateral pinning to correct supracondylar humeral fractures.

One disadvantage to using lateral pinning is that it is biomechanically inferior to cross pinning. Zionts et al. concluded that maximal stability was achieved using cross pinning after analyzing adult cadaveric specimens [10]. Zamzam and Bakarman found that type III fractures that were fixed with lateral only pinning with two pins were predisposed to postoperative instability, late complications and the need for a medial pin [11]. However with regard to torsional stability, Larson et al. found that there was no statistically significant difference between lateral pins versus cross pinning techniques in synthetic humeri [12]. Even with the biomechanical superiority of cross pinning, clinical outcomes in our study were the same. This may indicate the biomechanical superiority of cross pinning may not necessarily confer outcomes that are statistically or clinically significant. This sentence is "awkward" perhaps "this may indicate the biomechanical superiority of cross pinning may not necessarily confer outcomes that are either statistically or clinically significant” might be preferred. Also the authors might wish to state something about a power calculation here in terms of how many fractures needed to be treated in this way to identify a possible difference in outcome and how many fractures were not amenable to treatment with lateral wires only, owing to insufficient stability on stress-testing (stress testing as noted in methods section were 
done in all cases; in cases with persistent instability, all received medial pin. Therefore the number of medial pins placed is equal to number of persistent instability after stress testing).

The systematic review by Brauer et al. showed an increased risk of reduction loss using lateral pinning only compared to cross pinning [7]. We maintained adequate acceptable alignment without increasing need for reoperation. No fractures developed varus malalignment. Our results are similar to Lee et al. who performed lateral pinning for all fractures over a four-year period with excellent outcomes [3]. Kocher et al. also found excellent results with lateral only pinning in completely displaced type III fractures in a randomized trial [6]. Similar results were also obtained by Tripuraneni et al. as their prospective surgeon randomized trial showed no clinical difference between cross and lateral pinning [13]. Another prospective, surgeon randomized trial conducted by Gaston et al. also revealed that there was no statistical difference in radiographic outcomes of lateral vs. cross pinning of type III fractures [14]. Mahan et al. analyzed surgeon preference of cross pins vs. lateral only pinning after the prospective randomized control trial conducted by Kocher in 2007. Consistent with our result, Mahan et al. found that there was a statistically significant change in surgeon preference from cross pinning before the trial to lateral only pinning after the trial [15].

This study has several limitations. It is retrospective. It is possible that more unstable fractures required cross pinning. The study only evaluated two trauma surgeons at a level one trauma center and did not include data from other level two and three trauma centers. The study also did not evaluate any pediatric orthopedic surgeon preferences in pin placement. However, within a three year period-2009-20011-there was a significant reduction in the utilization of cross pinning with excellent clinic outcomes. Further limitations include the overall sample size and the lack of long-term clinical outcomes. Nevertheless, this study finds similar results as others in the recent literature about the reproducible outcomes with lateral pinning only for pediatric supracondylar humerus fractures.

Future studies analyzing trends in pin placement for supracondylar humeral fractures should include the comparison of academic to private orthopedic institutions. Additional studies can compare orthopedic trauma surgeon pin placement preference to pediatric orthopedic surgeon preference. The purpose of such comparisons would not only analyze trends within various health systems, but facilitate better communication between various orthopedic groups in treating supracondylar humeral fractures.

At a level one academic trauma center, there has been a trend toward decreasing cross pin fixation for pediatric supracondylar humerus fractures. Outcomes were excellent. We conclude that pediatric supracondylar fractures can be treated with lateral pin fixation only with excellent clinical outcomes.

\section{Conflict of Interest}

None.

\section{Ethical Statement}

The study was authorized by the local ethical committee and was performed in accordance with the Ethical standards of the 1964 Declaration of Helsinki as revised in 2000. This was a retrospective chart and radiographic review. No patient consent was applicable or obtained.

\section{Disclosures}

The authors have nothing to disclose.

\section{References}

[1] Houshian, S., Mehdi, B. and Larsen, M.S. (2001) The Epidemiology of Elbow Fracture in Children: Analysis of 355 Fractures, with Special Reference to Supracondylar Humerus Fractures. Journal of Orthopaedic Science, 6, 312-315. http://dx.doi.org/10.1007/s007760100024

[2] Mahan, S.T., May, C.D. and Kocher, M.S. (2007) Operative Management of Displaced Flexion Supracondylar Fractures in Children. Journal of Pediatric Orthopaedics, 27, 551-556. http://dx.doi.org/10.1097/01.bpb.0000279032.04892.6c

[3] Lee, Y.H., et al. (2008) Three Lateral Divergent or Parallel Pin Fixations for the Treatment of Displaced Supracondylar Humerus Fractures in Children. Journal of Pediatric Orthopaedics, 28, 417-422. 
http://dx.doi.org/10.1097/BPO.0b013e318173e13d

[4] Kim, W.Y., Chandru, R., Bonshahi, A., et al. (2003) Displaced Supracondylar Humeral Fractures in Children: Results of a National Survey of Pediatric Orthopedic Consultants. Injury, 34, 274-277. http://dx.doi.org/10.1016/S0020-1383(02)00321-2

[5] Lee, S.S., Mahar, A.T., Miesen, D., et al. (2002) Displaced Pediatric Supracondylar Humerus Fractures: Biomechanical Analysis of Percutaneous Pinning Techniques. Journal of Pediatric Orthopaedics, 22, 440-443. http://dx.doi.org/10.1097/01241398-200207000-00005

[6] Kocher, M.S., Kasser, J.R., Waters, P.M., et al. (2007) Lateral Entry Compared with Medial and Lateral Entry Pin Fixation for Completely Displaced Supracondylar Humeral Fractures in Children. A Randomized Control Trial. The Journal of Bone \& Joint Surgery, 89, 706-712. http://dx.doi.org/10.2106/JBJS.F.00379

[7] Brauer, C.A., Lee, B.M., Bae, D.S., et al. (2007) A Systematic Review of Medial and Lateral Entry Pinning Versus Lateral Entry Pinning for Supracondylar Fractures of the Humerus. Journal of Pediatric Orthopaedics, 27, 181-186. http://dx.doi.org/10.1097/bpo.0b013e3180316cf1

[8] Brown, I.C. and Zinar, D.M. (1995) Traumatic and Iatrogenic Neurological Complications after Supracondylar Humerus Fractures in Children. Journal of Pediatric Orthopaedics, 14, 440-443. http://dx.doi.org/10.1097/01241398-199507000-00005

[9] Bronwyn, S.L., Jackman, H., Tennant, S., et al. (2010) Iatrogenic Ulnar Nerve Injury after the Surgical Treatment of Displaced Supracondylar Fractures of the Humerus: Number Needed to Harm, A Systematic Review. Journal of Pediatric Orthopaedics, 30, 430-436. http://dx.doi.org/10.1097/BPO.0b013e3181e00c0d

[10] Zionts, L.E., McKellop, H.A. and Hathaway, R. (1994) Torsional Strength of Pin Configurations Used to Fix Supracondylar Fractures of the Humerus in Children. The Journal of Bone \& Joint Surgery, 76, 253-256.

[11] Zamzam, M.M. and Bakarman, K.A. (2009) Treatment of Displaced Supracondylar Humerus Fractures among Children: Crossed versus Lateral Pinning. Injury, 40, 625-630. http://dx.doi.org/10.1016/j.injury.2008.10.029

[12] Larson, L., Firoozbakhsh, K., Passarelli, R. and Bosch, P. (2006) Biomechanical Analysis of Pinning Techniques for Pediatric Supracondylar Humerus Fractures. Journal of Pediatric Orthopaedics, 26, 573-578. http://dx.doi.org/10.1097/01.bpo.0000230336.26652.1c

[13] Tripuraneni, K.R., Bosch, P.P., Schwend, R.M. and Yaste, J.J. (2009) Prospective, Surgeon-Randomized Evaluation of Crossed Pins versus Lateral Pins for Unstable Supracondylar Humerus Fractures in Children. Journal of Pediatric Orthopaedics Part B, 18, 93-98.

[14] Gaston, R.G., Cates, T.B., Devito, D., et al. (2010) Medial and Lateral Pin Versus Lateral-Entry Pin Fixation for Type 3 Supracondylar Fractures in Children: A Prospective, Surgeon-Randomized Study. Journal of Pediatric Orthopaedics, 30, 799-806. http://dx.doi.org/10.1097/BPO.0b013e3181f73d59

[15] Mahan, S.T., Osborn, E., Bae, D.S., et al. (2012) Changing Practice Patterns: The Impact of a RandomizedClinical Trial on Surgeons Preference for Treatmentof Type 3 Supracondylar Humerus Fractures. Journal of Pediatric Orthopaedics, 32, 340-345. http://dx.doi.org/10.1097/BPO.0b013e3182519d1d 